SUPPORTING INFORMATION

for the

communication

entitled

\title{
Highly Stereoselective [4 + 3] Cycloadditions of Nitrogen-Stabilized Oxyallyl Cations with Pyrroles. An Approach to Parvineostemonine.
}

\author{
authored by \\ Jennifer E. Antoline, Richard P. Hsung*, Jian Huang, Zhenlei Song, and Gang Li \\ Division of Pharmaceutical Sciences and Department of Chemistry \\ 7111 Rennebohm Hall, 777 Highland Avenue \\ University of Wisconsin, Madison, WI 53705
}


General Procedure for the [4 + 3] Cycloaddition of Pyrrole.

To a solution of the appropriate allenamide in $\mathrm{CH}_{2} \mathrm{Cl}_{2}[0.10 \mathrm{M}]$ was added 4 equiv of the appropriate pyrrole derivative and 2.0 equiv of $\mathrm{ZnCl}_{2}\left[1.0 \mathrm{M}\right.$ in $\left.\mathrm{Et}_{2} \mathrm{O}\right]$ and $4 \AA$ pulverized molecular sieves $[0.50 \mathrm{~g}]$. The reaction solution was cooled to $-45^{\circ} \mathrm{C}$, and 6.0 equiv of DMDO in acetone was added as a chilled solution [at $-78^{\circ} \mathrm{C}$ ] via a syringe pump over 2-3 $\mathrm{h}$. The syringe pump was cooled via wrapping of dry ice at all time during the addition. After the addition, the reaction mixture was stirred for another $2 \mathrm{~d}$. The reaction was then quenched with sat aq $\mathrm{NaHCO}_{3}$, filtered through Celite ${ }^{\mathrm{TM}}$, concentrated in vacuo, partitioned with $\mathrm{CH}_{2} \mathrm{Cl}_{2}$, extracted $\left[4\right.$ x $20 \mathrm{~mL}$ ], dried over $\mathrm{Na}_{2} \mathrm{SO}_{4}$, and concentrated in vacuo. The crude residue was purified via silica gel flash column chromatography [gradient eluent: $0 \%$ to $50 \%$ EtOAc in hexanes].

\section{Cycloadduct 7.}

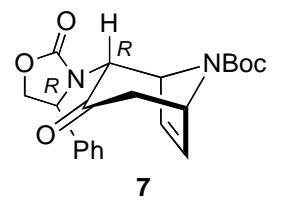

$\mathrm{R}_{f}=0.48[50 \%$ EtOAc in hexanes $]$;

${ }^{1} \mathrm{H}$ NMR (400 MHz, CDCl $) \delta$ 1.44-1.55 (m, 9H), 2.45 (d, $\left.J=16.8 \mathrm{~Hz}, 1 \mathrm{H}\right), 2.86$ (brd, 1H), 4.00 (brd, $0.5 \mathrm{H}, J=4.0 \mathrm{~Hz}), 4.09$ (br, 1H), 4.50 (br, 0.5H), 4.71 (br, 1H), 4.84-4.96 (br, 1.5H), 5.99 (br, 0.5H), 6.09 (br, $1 \mathrm{H}), 6.17(\mathrm{dd}, 1 \mathrm{H}, J=1.0,6.0 \mathrm{~Hz}), 7.06(\mathrm{dd}, 1 \mathrm{H}, J=2.0,6.0 \mathrm{~Hz}), 7.27-7.47(\mathrm{~m}, 5 \mathrm{H}) ;{ }^{13} \mathrm{C}$ NMR $(75$ $\left.\mathrm{MHz}, \mathrm{CDCl}_{3}\right) \delta 27.9,44.4,56.5,57.7,59.2,59.4,66.0,71.0,110.2,126.2,129.4,132.2,134.9,140.1$, 151.6, 159.1, 201.0; IR (neat) $\mathrm{cm}^{-1} 3064 \mathrm{w}, 2963 \mathrm{~s}, 1777 \mathrm{~s}, 1725 \mathrm{~s}, 1416 \mathrm{~s}$; mass spectrum (APCI): m/e (\% relative intensity) $407(60)(\mathrm{M}+\mathrm{Na})^{+} ; \mathrm{m} / \mathrm{e}$ calcd for $\mathrm{C}_{21} \mathrm{H}_{24} \mathrm{~N}_{2} \mathrm{O}_{5} \mathrm{Na} 407.1577$, found 407.1561.

\section{Cycloadduct 8.}

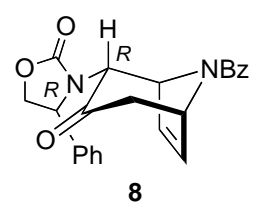

$\mathrm{R}_{f}=0.35[50 \%$ EtOAc in hexanes];

${ }^{1} \mathrm{H}$ NMR (500 MHz, $\mathrm{CDCl}_{3}$ ) $\delta$ 2.29-2.75 (brm, 3H), 2.95-3.01 (brm, 1H), 3.49-3.54 (brm, 1H), 3.92 (br, 0.3H), 4.10-4.16 (brm, 1H), 4.61-4.83 (brm, 4H), 4.94-5.11 (brm, 4H), 4.94-5.11 (brm, 1.7H), 5.25-5.29 (brm, 1H), 5.41 (brs, 1H), 5.66 (brs, 0.5H), 5.81 (brs, 0.5H), 6.05-6.50 (brm, 4H), 7.12-7.22 (br, 2H), 7.34-7.59 (br, 8H) (extra resonances due to rotamers); IR (neat) $\mathrm{cm}^{-1} 3057 \mathrm{w}, 2981 \mathrm{w}, 1756 \mathrm{~s}, 1697 \mathrm{~s}$, $1455 \mathrm{~m}$; mass spectrum (APCI): $\mathrm{m} / \mathrm{e}\left(\%\right.$ relative intensity) $411(92)(\mathrm{M}+\mathrm{Na})^{+} ; \mathrm{m} / \mathrm{e}$ calcd for $\mathrm{C}_{23} \mathrm{H}_{20} \mathrm{~N}_{2} \mathrm{O}_{4} \mathrm{Na}$ 411.1315, found 411.1297. 


\section{Cycloadduct 9.}

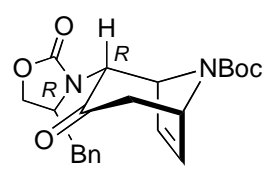

9: $88 \%[d r: 88: 12]$

$\mathrm{R}_{f}=0.46[50 \%$ EtOAc in hexanes];

${ }^{1} \mathrm{H}$ NMR (500 MHz, $\left.\mathrm{CDCl}_{3}\right) \delta 1.44(\mathrm{br}, 9 \mathrm{H}), 2.46(\mathrm{dd}, 1 \mathrm{H}, J=8.5,8.5 \mathrm{~Hz}), 2.53-2.95$ (br, 1.5H), 2.973.30 (br, 1H), 3.87-4.06 (brm, 1.5H), 4.08-4.28 (br, 1.5H), 5.47 (br, 1H), 5.71-5.84 (br, 1.5H), 5.86-6.10 (brm, 1.5H), 6.16-6.41 (brm, 1.5H), 6.99-7.31 (brm, 5H); $\left.{ }^{13} \mathrm{C} \mathrm{NMR} \mathrm{(75} \mathrm{MHz,} \mathrm{CDCl}_{3}\right) \delta 27.9$, 39.3, 44.2. 56.1, 59.8, 65.3, 67.3, 81.2, 83.8, 84.3, 86.2, 101.5, 127.2, 128.9, 131.9, 135.2, 151.8, 201.0; IR (neat) $\mathrm{cm}^{-1} 2978 \mathrm{w}, 2929 \mathrm{w}, 1760 \mathrm{~s}, 1721 \mathrm{~s}, 1395 \mathrm{~m}, 1368 \mathrm{~m}$; mass spectrum (APCI): m/e (\% relative intensity) $421(80)(\mathrm{M}+\mathrm{Na})^{+} ; \mathrm{m} / \mathrm{e}$ calcd for $\mathrm{C}_{22} \mathrm{H}_{26} \mathrm{~N}_{2} \mathrm{O}_{5} \mathrm{Na} 421.1739$, found 421.1748 .

\section{Cycloadduct 10.}

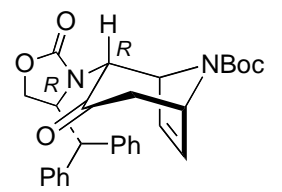

10: $70 \%[d r: 78: 22]$

$\mathrm{R}_{f}=0.47[50 \%$ EtOAc in hexanes $] ;[\alpha]^{23}{ }_{\mathrm{D}}=-18.8\left(\mathrm{c}=0.17, \mathrm{CH}_{2} \mathrm{Cl}_{2}\right)$;

${ }^{1} \mathrm{H}$ NMR (500 MHz, $\mathrm{CDCl}_{3}$ ) $\delta$ 1.33-1.62 (m, 9H), 2.38-2.54 (br, 2H), 3.72-3.82 (br, 1H), 4.02-4.10 (br, $1 \mathrm{H}), 4.22-4.52(\mathrm{~m}, 1 \mathrm{H}), 4.36-4.48(\mathrm{~m}, 3 \mathrm{H}), 4.70-4.80(\mathrm{~m}, 1 \mathrm{H}), 6.08-6.22(\mathrm{br}, 1 \mathrm{H}), 6.41(\mathrm{dd}, 1 \mathrm{H}, J=3.0$, $3.0 \mathrm{~Hz}), 7.18-7.44(\mathrm{~m}, 10 \mathrm{H}) ;{ }^{13} \mathrm{C} \mathrm{NMR}\left(75 \mathrm{MHz}, \mathrm{CDCl}_{3}\right) \delta 14.0,27.8,27.9,28.0,28.3,44.6,51.5,60.3$, $82.9,125.0,127.4,127.7,127.8,128.3,128.9,129.3,134.7,140.3,145.0,150.3,151.5,201.4$ (extra resonances due to rotamers); IR (neat) $\mathrm{cm}^{-1} 2980 \mathrm{~m}, 2936 \mathrm{w}, 1764 \mathrm{~s}, 1725 \mathrm{~s}, 1367 \mathrm{~s}$; mass spectrum (ACPI): m/e (\% relative intensity) 375 (100) $(\mathrm{M}+\mathrm{H}-\mathrm{BOC})^{+}, 308$ (58); m/e calcd for $\mathrm{C}_{28} \mathrm{H}_{30} \mathrm{~N}_{2} \mathrm{O}_{5} \mathrm{Na}$ 497.2047, found 497.2056.

\section{Cycloadduct 11.}

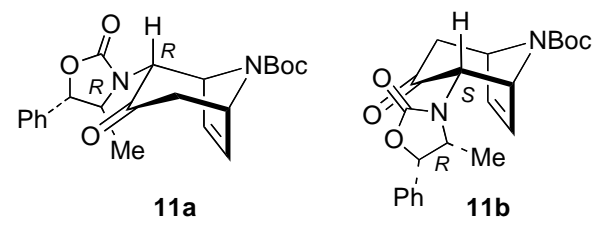

Isomer-a: $\mathrm{R}_{f}=0.42[50 \% \mathrm{EtOAc} /$ hexane $] ; \operatorname{mp} 187-191{ }^{\circ} \mathrm{C} ;[\alpha]^{23} \mathrm{D}=-22.9\left(\mathrm{c}=0.18, \mathrm{CH}_{2} \mathrm{Cl}_{2}\right)$;

${ }^{1} \mathrm{H}$ NMR $\left(500 \mathrm{MHz} \mathrm{CDCl}_{3}\right) \delta 0.78(\mathrm{~d}, 3 \mathrm{H}, J=7.0 \mathrm{~Hz}), 1.53$ (br, 9H), 2.54 (brd, 1H), $2.73(\mathrm{brd}, 0.5 \mathrm{H})$, 2.91 (brd, 0.5H), 3.96 (dt, 1H, $J=6.5,7.0 \mathrm{~Hz}), 4.36$ (br, 0.5H), 4.61 (br, 0.5H), 4.79-4.96 (brm, 2H), 5.77 (brd, 1H), 6.23-6.37 (brm, 2H), 7.32-7.36 (brm, 3H), 7.38-7.41 (brm, 2H); IR (neat) cm-1 2974w, $1760 \mathrm{~s}, 1721 \mathrm{~m}, 1698 \mathrm{~s}, 1413 \mathrm{~m}$; mass spectrum (APCI): m/e (\% relative intensity) 299.2 (100) $(\mathrm{M}+\mathrm{H}-$ 
Boc) $)^{+} ; \mathrm{m} / \mathrm{e}(\mathrm{MALDI})$ calcd for $\mathrm{C}_{22} \mathrm{H}_{26} \mathrm{~N}_{2} \mathrm{O}_{5} \mathrm{Na}^{+} 421.1733$, found 421.1728 .

Isomer-b: $\mathrm{R}_{f}=0.35[50 \% \mathrm{EtOAc} /$ hexane $] ;[\alpha]^{23}{ }_{\mathrm{D}}=18.5\left(\mathrm{c}=0.34, \mathrm{CH}_{2} \mathrm{Cl}_{2}\right)$;

${ }^{1} \mathrm{H} \mathrm{NMR}\left(500 \mathrm{MHz} \mathrm{CDCl}_{3}\right) \delta 0.82$ (brd, 3H), 1.50 (s, 9H), 2.52 (brm, 1H), 2.72 (brd, 0.5H), 2.88 (brd, $0.5 \mathrm{H}), 3.46$ (br, 0.5H), 4.14 (br, 1.5H), 4.35 (br, 0.25H), 4.74-4.84 (brm, 1.5H), 5.04 (br, 0.25H), 5.70 (br, $1 \mathrm{H}), 6.16(\mathrm{br}, 0.2 \mathrm{H})$ and $6.31(\mathrm{br}, 0.8 \mathrm{H}), 6.45$ (br, 1H), 7.28-7.30 (brm, 2H), 7.38-7.41 (brm, 3H); IR (neat) $\mathrm{cm}^{-1} 2974 \mathrm{w}, 1751 \mathrm{~s}, 1724 \mathrm{~m}, 1694 \mathrm{~s}, 1408 \mathrm{~m}, 1367 \mathrm{~m}$; mass spectrum (APCI): $\mathrm{m} / \mathrm{e}$ (\% relative intensity) 299.2 (100) $(\mathrm{M}+\mathrm{H}-\text { Boc })^{+} ; \mathrm{m} / \mathrm{e}$ (MALDI) calcd for $\mathrm{C}_{22} \mathrm{H}_{26} \mathrm{~N}_{2} \mathrm{O}_{5} \mathrm{Na} 421.1733$, found 421.1728 .

\section{Cycloadduct 12.}

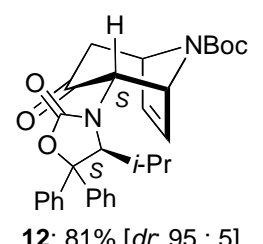

$\mathrm{R}_{\mathrm{f}}=0.27[33 \%$ EtOAc in hexanes $) ;[\alpha]^{23} \mathrm{D}=-131.0\left(\mathrm{c}=0.40, \mathrm{CH}_{2} \mathrm{Cl}_{2}\right)$;

${ }^{1} \mathrm{H}$ NMR $\left(300 \mathrm{MHz}, \mathrm{CDCl}_{3}\right) \delta$ 0.75-0.83 (m, 3H), 1.13-1.34 (m, 3H), $1.50(\mathrm{~s}, 9 \mathrm{H}), 1.96-2.12(\mathrm{~m}, 1 \mathrm{H})$, 2.38-2.52 (m, 1H), 2.54-2.82 (m, 1H), 3.91-4.10 (br, 1H), 4.35-4.52 (br, 1H), 4.60-4.88 (br, 1H), 5.045.18 (br, $1 \mathrm{H}), 6.16-6.30$ (br, $1 \mathrm{H}), 6.44-6.64$ (br, $1 \mathrm{H}), 7.21-7.56(\mathrm{~m}, 8 \mathrm{H}), 7.57-7.68(\mathrm{~m}, 2 \mathrm{H}) ;{ }^{13} \mathrm{C} \mathrm{NMR}$ $\left(75 \mathrm{MHz}, \mathrm{CDCl}_{3}\right) \delta 15.2,15.3,15.5,20.7,21.5,22.1,27.8,27.9,28.2,28.3,29.4,44.4,44.7,51.4,56.0$, $56.5,60.4,60.5,65.5,67.4,68.6,71.9,72.6,80.7,81.2,87.9,124.7,125.3,125.5,126.1,126.3,127.5$, 128.0, 128.3, 128.4, 131.8, 132.7, 133.8, 134.0, 139.0, 139.1, 144.1, 144.3, 151.1, 151.8, 156.2, 199.0, 199.2 (extra resonances due to rotamers); IR (neat) $\mathrm{cm}^{-1} 2972 \mathrm{~m}, 2968 \mathrm{~m}, 1752 \mathrm{~s}, 1707 \mathrm{~s}, 1372 \mathrm{~s}$; mass spectrum (ACPI): m/e (\% relative intensity) 403 (92) (M + H - BOC) ${ }^{+}, 359$ (40), 292 (100), 238 (38), 167 (30); m/e calcd for $\mathrm{C}_{30} \mathrm{H}_{34} \mathrm{~N}_{2} \mathrm{O}_{5} \mathrm{Na} 525.2360$, found 525.2361.

\section{Cycloadduct 13.}

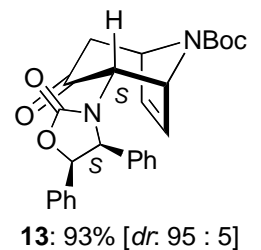

$\mathrm{R}_{f}=0.55[50 \%$ EtOAc in hexanes $] ;[\alpha]^{23}=34.6\left(\mathrm{c}=2.00, \mathrm{CH}_{2} \mathrm{Cl}_{2}\right)$;

${ }^{1} \mathrm{H}$ NMR $\left(500 \mathrm{MHz}, \mathrm{CDCl}_{3}\right) \delta 1.49-1.60(\mathrm{~m}, 9 \mathrm{H}), 2.50(\mathrm{brd}, 1 \mathrm{H}), 2.78-2.94(\mathrm{~m}, 1 \mathrm{H}), 4.43(\mathrm{br}, 0.5 \mathrm{H})$, 4.66-4.80 (br, 1.5H), 4.91 (br, 0.5H), 4.97-5.05 (br, 1.5H), 5.19-5.23 (br, 0.5H), 5.96 (d, 1H, J = 7.5 $\mathrm{Hz}), 6.04(\mathrm{br}, 1 \mathrm{H}), 6.55$ (br, 0.25H), $6.18(\mathrm{br}, 0.25 \mathrm{H}), 6.89-7.19(\mathrm{~m}, 10 \mathrm{H}) ;{ }^{13} \mathrm{C} \mathrm{NMR}\left(75 \mathrm{MHz}, \mathrm{CDCl}_{3}\right) \delta$ $13.9,27.9,44.5,51.5,56.4,59.6,62.3,66.2$, 79.2, 81.5, 126.1,127.7, 127.9, 128.4, 132.9, 134.2, 135.6, 
145.1, 146.5, 158.8, 201.5; IR (neat) $\mathrm{cm}^{-1} 3064 \mathrm{w}, 2963 \mathrm{~s}, 1777 \mathrm{~s}, 1725 \mathrm{~s}, 1416 \mathrm{~s}$; mass spectrum (APCI): $\mathrm{m} / \mathrm{e}\left(\%\right.$ relative intensity) $483(83)(\mathrm{M}+\mathrm{Na})^{+} ; \mathrm{m} / \mathrm{e}$ calcd for $\mathrm{C}_{27} \mathrm{H}_{28} \mathrm{~N}_{2} \mathrm{O}_{5} \mathrm{Na} 483.1890$, found 483.1906 .

\section{Cycloadduct 14.}

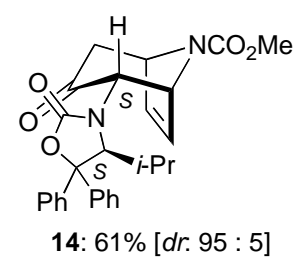

$\mathrm{R}_{f}=0.30[50 \%$ EtOAc in hexanes $] ;[\alpha]^{23}{ }_{\mathrm{D}}=-81.3\left(\mathrm{c}=0.15, \mathrm{CH}_{2} \mathrm{Cl}_{2}\right)$;

${ }^{1} \mathrm{H}$ NMR $\left(500 \mathrm{MHz}, \mathrm{CDCl}_{3}\right) \delta$ 0.67-0.69 (br, 1H), 0.67-0.70 (br, 1H), 0.73-0.78 (br, 1H), 0.86-0.89 (m, $1 \mathrm{H}), 1.11-1.16(\mathrm{~m}, 1 \mathrm{H}), 1.2-1.24(\mathrm{~m}, 1 \mathrm{H}), 1.85-1.93(\mathrm{~m}, 1 \mathrm{H}), 1.97-2.05(\mathrm{~m}, 1 \mathrm{H}), 2.4-2.46(\mathrm{~m}, 1 \mathrm{H}), 2.54-$ $2.61(\mathrm{~m}, 1 \mathrm{H}), 2.68-2.75(\mathrm{~m}, 1 \mathrm{H}), 3.77(\mathrm{~s}, 3 \mathrm{H}), 3.89-3.93(\mathrm{br}, 0.5 \mathrm{H}), 4.01-4.05(\mathrm{br}, 0.5 \mathrm{H}), 4.33-4.40(\mathrm{~m}$, $1 \mathrm{H}), 4.70-4.75(\mathrm{br}, 0.5 \mathrm{H}), 4.79-4.83(\mathrm{br}, 0.5 \mathrm{H}), 5.08-5.12$ (br, 0.5H), 5.21-5.26 (br, 0.5H), 6.16-6.22 (br, 1H), 6.51-6.60 (br, 1H), 7.21-7.45 (m, 8H), 7.54-7.59 (m, 2H); ${ }^{13} \mathrm{C}$ NMR (75 MHz, $\left.\mathrm{CDCl}_{3}\right) \delta 15.1,15.4$, 20.6, 21.4, 29.4, 44.4, 44.9, 51.5, 52.7, 53.5, 56.4, 60.8, 65.6, 66.6, 67.8, 68.7, 72.4, 72.8, 88.1, 89.2, $124.8,125.2,125.6,126.2,127.4,127.6,128.0,128.2,128.4,128.8,129.0,131.7,132.1,133.7,139.1$, 143.8, 144.4, 145.8, 152.3, 152.9, 156.1, 158.5, 198.2 (extra resonances due to rotamers); IR (neat) $\mathrm{cm}^{-1}$ $2960 \mathrm{~m}, 1755 \mathrm{~s}, 1747 \mathrm{~s}, 1450 \mathrm{~s}$; mass spectrum (ACPI): m/e (\% relative intensity) $451(12)(\mathrm{M}+\mathrm{H})^{+} ; \mathrm{m} / \mathrm{e}$ calcd for $\mathrm{C}_{27} \mathrm{H}_{28} \mathrm{~N}_{2} \mathrm{O}_{5} \mathrm{Na} 483.1890$, found 483.1897 .

\section{Cycloadduct 15.}

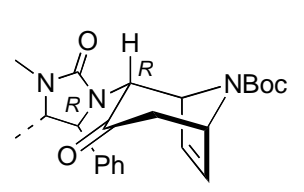

15: $35 \%[d r: 95: 5]$

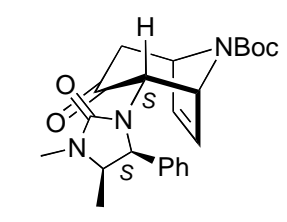

ent-15: 30\% [dr: $95: 5]$

$\mathrm{R}_{f}=0.43[60 \%$ EtOAc in hexanes $] ;[\alpha]^{23}=-77.5\left(\mathrm{c}=0.1, \mathrm{CH}_{2} \mathrm{Cl}_{2}\right)$;

${ }^{1} \mathrm{H}$ NMR $\left(500 \mathrm{MHz}, \mathrm{CDCl}_{3}\right) \delta 0.72(\mathrm{~d}, 3 \mathrm{H}, J=7.5 \mathrm{~Hz}), 1.24-1.29(\mathrm{brm}, 9 \mathrm{H}), 2.40(\mathrm{~d}, 1 \mathrm{H}, J=17.5 \mathrm{~Hz})$, $2.74(\mathrm{~s}, 3 \mathrm{H}), 2.85(\mathrm{~d}, 1 \mathrm{H}, J=14.5 \mathrm{~Hz}), 3.98(\mathrm{dq}, 1 \mathrm{H}, J=7.5,4.0 \mathrm{~Hz}, 1 \mathrm{H}), 4.33-4.71$ (brm, 2.5H), 5.02 (br, 0.5H), 5.07 (d, 1H, J=3.9 Hz), 5.21-5.35 (brm, 1H), 6.06 (dd, 1H, J = 2.4, 6.0 Hz) 7.28-7.44 (brm, $5 \mathrm{H}) ;{ }^{13} \mathrm{C} \mathrm{NMR}\left(125 \mathrm{MHz}, \mathrm{CDCl}_{3}\right) \delta 15.3,28.3,29.4,44.6,56.7,57.2,59.9,61.5,66.1,81.6,127.3$, $128.5,129.3,132.9,134.9,138.7,152.3,162.4,203.3$; IR (neat) $\mathrm{cm}^{-1} 3055 \mathrm{w}, 2853 \mathrm{w}, 1746 \mathrm{~s}, 1691 \mathrm{~s}$; mass spectrum (APCI): m/e (\% relative intensity) $434(100)(\mathrm{M}+\mathrm{Na})^{+} ; \mathrm{m} / \mathrm{e}$ calcd for $\mathrm{C}_{23} \mathrm{H}_{29} \mathrm{~N}_{3} \mathrm{O}_{4} \mathrm{Na}$ 434.2050, found 434.2061.

\section{Cycloadduct ent-15.}

$[\alpha]^{23}{ }_{\mathrm{D}}=+135.4\left(\mathrm{c} 0.30, \mathrm{CH}_{2} \mathrm{Cl}_{2}\right)$. 
REMOVAl OF THE OXAZOLIDINONE AUXILIARY.

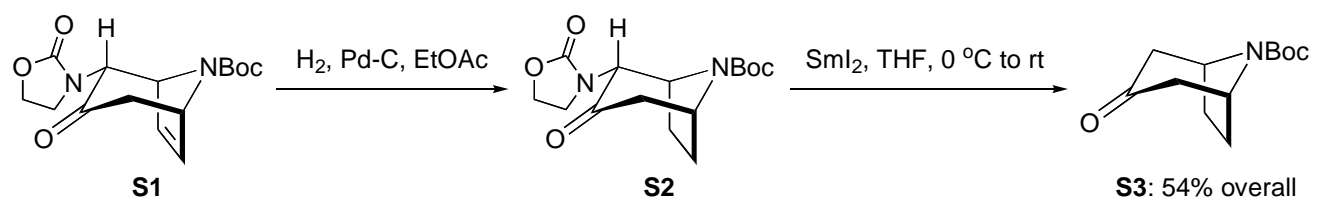

Cycloadduct S1. $\mathrm{R}_{f}=0.23\left[50 \%\right.$ EtOAc in hexanes]; ${ }^{1} \mathrm{H}$ NMR $\left(400 \mathrm{MHz}, \mathrm{CDCl}_{3}\right) \delta 1.53(\mathrm{br}$, 9H), 2.48 (d, 0.8H, $J=16.0 \mathrm{~Hz}$ ), 2.67-2.98 (brm, 1.2H), 3.24 (br, 0.2H), 3.41 (br, 0.8H), 3.51-3.84 (br, $1 \mathrm{H}), 4.38(\mathrm{~m}, 1 \mathrm{H}), 4.74-5.06$ (brm, 2H) 5.66-6.48 (brm, 4H); ${ }^{13} \mathrm{C} \mathrm{NMR}\left(100 \mathrm{MHz}, \mathrm{CDCl}_{3}\right) \delta 28.5,42.9$, $62.7,65.5,68.2,81.7,85.0,101.7,131.8,136.7,153.5,158.9,201.2$; IR (neat) $\mathrm{cm}^{-1} 3100 \mathrm{w}, 3250 \mathrm{w}$, $1793 \mathrm{~s}, 1697 \mathrm{~s}, 1493 \mathrm{~m}$; mass spectrum $(\mathrm{APCI}): \mathrm{m} / \mathrm{e}\left(\%\right.$ relative intensity) $331(100)(\mathrm{M}+\mathrm{Na})^{+}$.

Hydrogenation. To a solution of cycloadduct $\mathbf{S 1}$ [44.0 $\mathrm{mg}, 0.15 \mathrm{mmol}$ ] in EtOAc [2 $\mathrm{mL}]$ at $\mathrm{rt}$ was added 5\% Pd-C [5.0 mg]. The heterogeneous mixture was stirred under 1 atm of $\mathrm{H}_{2}$ for $3 \mathrm{~h}$. Filtration through Celite ${ }^{\mathrm{TM}}$ led to quantitative isolation of $\mathbf{S} 2$.

S2: $\mathrm{R}_{f}=0.23\left[50 \%\right.$ EtOAc in hexanes]; ${ }^{1} \mathrm{H}$ NMR (400 MHz, $\left.\mathrm{CDCl}_{3}\right) \delta 1.53(\mathrm{br}, 10 \mathrm{H}), 1.67(\mathrm{br}$, $1 \mathrm{H}), 1.83$ (br, 1H), 2.24 (br, 2H), 2.44 (dd, 1H, $J=1.4,15.2 \mathrm{~Hz}), 2.80$ (br, 2H) 3.57(dq, 1H, $J=1.5,6.8$ $\mathrm{Hz}), 4.02$ (br, 1H), 4.37 (dt, $1 \mathrm{H}, J=1.5,9.2 \mathrm{~Hz}), 4.43$ (dt, $1 \mathrm{H}, J=1.5,9.2 \mathrm{~Hz}), 4.49-4.71(\mathrm{br}, 1 \mathrm{H}) ;{ }^{13} \mathrm{C}$ NMR $\left(100 \mathrm{MHz}, \mathrm{CDCl}_{3}\right) \delta 28.5,28.7,43.0,48.1,53.9,57.8,62.9,64.8,81.6,83.0,154.1,158.8,203.6$; IR (neat) $\mathrm{cm}^{-1} 2979 \mathrm{w}, 3250 \mathrm{w}, 1750 \mathrm{~s}, 1699 \mathrm{~s}$; mass spectrum (APCI): m/e (\% relative intensity) 333 (100) $(\mathrm{M}+\mathrm{Na})^{+}$.

Removal of Auxiliary. To a solution of the above hydrogenated cycloadduct S2 [13.0 mg, 0.043 $\mathrm{mmol}]$ in THF $[0.2 \mathrm{~mL}]$ at $0{ }^{\circ} \mathrm{C}$ was added a solution of freshly prepared $\mathrm{SmI}_{2}$ [2.2 $\mathrm{mL}, 0.10 \mathrm{M}, 0.22$ mmol] in THF. The reaction went from dark blue to yellow in $45 \mathrm{~min}$ and TLC indicated the reaction was done. $\mathrm{HCl}\left[\begin{array}{ll}1 & M\end{array}\right]$ was added then the mixture was filtered through celite. The aqueous layer was separated and extracted with ether [4 x $25 \mathrm{~mL}$ ], the combined organic layers were washed with equal volume sat aq $\mathrm{NaHCO}_{3}$, and $\mathrm{Na}_{2} \mathrm{~S}_{2} \mathrm{O}_{3}$, dried over $\mathrm{NaSO}_{4}$, and concentrated in vacuo. The crude oil was purified by preparative thin layer chromatography [50\% EtOAc in hexanes] to give S3 [54\%].

S3: $\mathrm{R}_{f}=0.77\left[50 \%\right.$ EtOAc in hexanes]; ${ }^{1} \mathrm{H} \mathrm{NMR}\left(500 \mathrm{MHz}, \mathrm{CDCl}_{3}\right) \delta 1.26(\mathrm{br}, 2 \mathrm{H}), 1.50$ (brs, 9H), 1.66 (brd, 1H), 2.09 (br, 1.5H), 2.32 (brd, 1.5H), 2.65 (brd, 2H), 4.45 (brd, 2H); IR (neat) $\mathrm{cm}^{-1}$ 2981w, 1756s, 1697s, 1455m; mass spectrum (APCI): m/e (\% relative intensity) $284(40)(\mathrm{M}+\mathrm{Na})^{+}$. 
TRANSFORMATIONS OF CYCLOADDUCT 13.

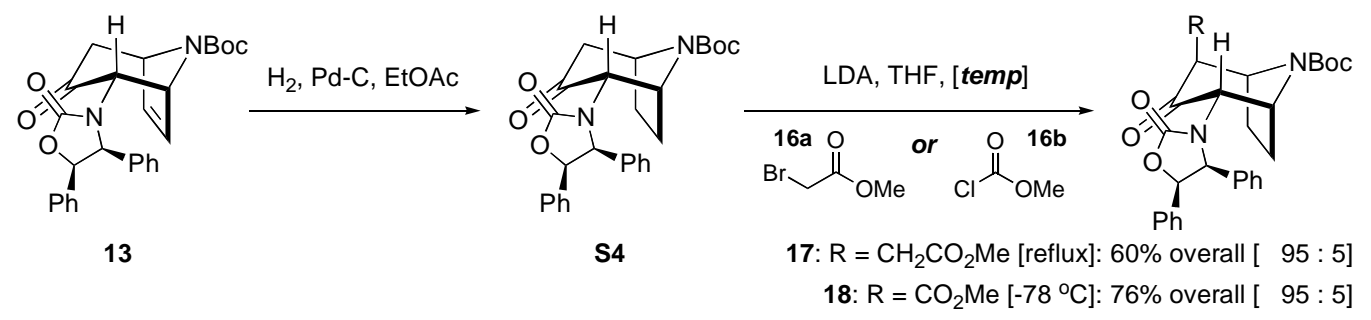

Hydrogenation. To a solution of 13 [250.0 mg, $0.56 \mathrm{mmol}$ ] in EtOAc [10 $\mathrm{mL}]$ at $\mathrm{rt}$ was added $5 \% \mathrm{Pd}-\mathrm{C}[50.0 \mathrm{mg}]$. The heterogeneous mixture was stirred under $1 \mathrm{~atm}$ of $\mathrm{H}_{2}$ for $3 \mathrm{~h}$. Filtration through Celite $^{\mathrm{TM}}$ led to quantitative isolation of S4.

S4: $\mathrm{R}_{f}=0.56\left[50 \%\right.$ EtOAc in hexanes]; $[\alpha]^{23}{ }_{\mathrm{D}}=-76.9\left(\mathrm{c}=1.20, \mathrm{CH}_{2} \mathrm{Cl}_{2}\right) ;{ }^{1} \mathrm{H} \mathrm{NMR}(400 \mathrm{MHz}$, $\left.\mathrm{CDCl}_{3}\right) \delta$ 1.27-1.74 (brm, $\left.11 \mathrm{H}\right), 1.79-2.10(\mathrm{br}, 1.5 \mathrm{H}), 2.26(\mathrm{~d}, 0.2 \mathrm{H}, J=14.7 \mathrm{~Hz}), 2.46(\mathrm{~d}, 0.8 \mathrm{H}, J=$ $14.7 \mathrm{~Hz}$ ), 2.58-3.04 (br, 2H), 3.14 (br, 0.1H), 3.31 (br, 0.2H), 3.43 (br, 0.2H), 3.83 (br, 0.2H), 4.11-4.50 (br, 1H), 4.53 (br, 0.1H), 4.68 (br, 0.1H), 4.81 (brd, 0.9H, J = 3.6 Hz), 5.16 (br, 0.5H), 5.55(br, 0.2H), $5.92(\mathrm{~d}, 0.3 \mathrm{H}, J=8.0 \mathrm{~Hz}), 6.02(\mathrm{~d}, 0.7 \mathrm{H}, J=8.0 \mathrm{~Hz}), 6.95-7.25(\mathrm{brm}, 10 \mathrm{H})$; IR (neat) cm $\mathrm{cm}^{-1} 2977 \mathrm{w}$, $1757 \mathrm{~s}, 1723 \mathrm{~m}, 1697 \mathrm{~s}, 1394 \mathrm{~m}$; mass spectrum $(\mathrm{EI}): \mathrm{m} / \mathrm{e}\left(\%\right.$ relative intensity) $485(55)(\mathrm{M}+\mathrm{Na})^{+} ; \mathrm{m} / \mathrm{e}$ calcd for $\mathrm{C}_{22} \mathrm{H}_{22} \mathrm{~N}_{2} \mathrm{O}_{3}(\mathrm{M}-\mathrm{Boc}+\mathrm{H}) 362.1625$, found 362.1696 .

Alkylation of S4. Diisopropyl amine [0.09 $\mathrm{mL}, 0.67 \mathrm{mmol}]$ was dissolved in THF [0.70 $\mathrm{mL}]$, cooled to $-78{ }^{\circ} \mathrm{C}$. After which, $n$-BuLi $[0.33 \mathrm{~mL}, 2.0 \mathrm{M}, 0.67 \mathrm{mmol}]$ was added and the resulting mixture was stirred for $30 \mathrm{~min}$ before being warmed to $0{ }^{\circ} \mathrm{C}$ and stirred for an additional $30 \mathrm{~min}$. The solution was cooled back down to $-78{ }^{\circ} \mathrm{C}$ and a solution of compound S4 [0.20 g, $\left.0.45 \mathrm{mmol}\right]$ in THF $[0.7 \mathrm{~mL}]$ was added dropwise and the resulting mixture was stirred at $-78{ }^{\circ} \mathrm{C}$ for $1 \mathrm{~h}$ before methyl $\alpha$ bromoacetate $[0.045 \mathrm{~mL}, 0.47 \mathrm{mmol}]$ was added dropwise. The reaction mixture was allowed to stir for $4 \mathrm{~h}$ before being warmed to $\mathrm{rt}$ and heated to reflux for $6 \mathrm{~h}$. The mixture was quenched with sat aq $\mathrm{NaHCO}_{3}$, extracted with EtOAc, dried with $\mathrm{Na}_{2} \mathrm{SO}_{4}$, filtered, and concentrated in vacuo. The crude residue was purified via silica gel flash column chromatography [gradient eluent: $0 \%$ to $50 \%$ EtOAc in hexanes] to give ester 17 [60\%] as yellow oil.

Ester 17: $\mathrm{R}_{f}=0.61\left[50 \%\right.$ EtOAc in hexanes]; $[\alpha]^{23}{ }_{\mathrm{D}}=33.6\left(\mathrm{c}=0.60, \mathrm{CH}_{2} \mathrm{Cl}_{2}\right) ;{ }^{1} \mathrm{H}$ NMR $(500$ $\mathrm{MHz}, \mathrm{CDCl}_{3}$ ) $\delta 1.37$ (br, 9H), 1.41-1.58 (brm, 2H), 1.78-2.03 (brm, 2H), 2.88-2.98 (br, 1H), 3.63-3.85 (brm, 3H), 4.01-4.27 (brm, 2.5H), 4.28-4.42 (brm, 1.5H), 4.43-4.59 (brm, 1H), 5.34-5.59 (brm, 1H), $5.97(\mathrm{~d}, 1 \mathrm{H}, J=8.0 \mathrm{~Hz}), 6.89-7.16$ (brm, $10 \mathrm{H}) ;{ }^{13} \mathrm{C} \mathrm{NMR}\left(125 \mathrm{MHz}, \mathrm{CDCl}_{3}\right) \delta$ 14.2, 19.4, 28.4, 29.5, $50.2,52.4,53.7,54.1,65.0,69.8,74.8,80.1,98.4,119.4,126.3,127.9,128.2,128.7,135.2,145.7,156.0$, 169.7, 187.1, 211.1; IR (neat) $\mathrm{cm}^{-1} 2979,2963 \mathrm{~s}, 1776 \mathrm{~s}, 1693 \mathrm{~s}$; mass spectrum (APCI): m/e (\% relative intensity) 557 (100) $(\mathrm{M}+\mathrm{Na})^{+}$; m/e calcd for $\mathrm{C}_{30} \mathrm{H}_{34} \mathrm{~N}_{2} \mathrm{O}_{7} \mathrm{Na} 557.2258$, found 557.2244.

Acylation of S4. Diisopropyl amine [0.020 mL, $0.13 \mathrm{mmol}]$ was dissolved in THF [0.50 mL] and the solution was cooled to $-78{ }^{\circ} \mathrm{C}$. After which, $n$-BuLi $[0.05 \mathrm{~mL}, 2.2 \mathrm{M}, 0.12 \mathrm{mmol}]$ was added 
dropwise and the result solution was stirred for $30 \mathrm{~min}$ before being warmed up to $0{ }^{\circ} \mathrm{C}$ and stirred for an additional $30 \mathrm{~min}$. The solution was cooled back down to $-78{ }^{\circ} \mathrm{C}$ and a solution of compound S4 [50.0 $\mathrm{mg}, 0.11 \mathrm{mmol}]$ in THF $[0.5 \mathrm{~mL}]$ was added dropwise. The resulting mixture was stirred at $-78{ }^{\circ} \mathrm{C}$ for 1 $\mathrm{h}$ before methyl chloroformate $[0.15 \mathrm{~mL}, 0.13 \mathrm{mmol}]$ was added dropwise. The reaction mixture was allowed to stir for $12 \mathrm{~h}$ while slowly warming to $\mathrm{rt}$. The reaction was quenched with equal volume of sat aq $\mathrm{NaHCO}_{3}$, extracted with EtOAc [4 x $30 \mathrm{~mL}$ ], dried over $\mathrm{Na}_{2} \mathrm{SO}_{4}$, and concentrated in vacuo. The crude residue was purified via silica gel flash column chromatography [gradient eluent: $0 \%$ to $50 \%$ EtOAc in hexanes] to give $\beta$-ketoester 18 [76\%] as white foam.

$\beta$-Ketoester 18: $\mathrm{R}_{f}=0.60\left[50 \%\right.$ EtOAc in hexanes]; $[\alpha]^{23}{ }_{\mathrm{D}}=-24.0\left(\mathrm{c}=6.00, \mathrm{CH}_{2} \mathrm{Cl}_{2}\right) ;{ }^{1} \mathrm{H} \mathrm{NMR}$ $\left(500 \mathrm{MHz} \mathrm{CDCl}_{3}\right) \delta 1.01-1.24$ (brm, 9H), 1.25-1.55 (brm, 2H), 1.57-2.18 (brm, 1H), 2.28 (br, 0.75H), 2.44 (br, 0.25H), 2.79 (brm, 1H), 3.61 (s, 3H), 3.76 (brm, 2H), 4.00-5.09 (brm, 1H), 5.29 (d, 0.5H), 5.47 (br, 0.5H), 5.77-6.10 (br, 0.5H), 6.25-6.63 (br, 0.5H), 6.72-7.10 (brm, 8H), 7.11-7.34 (br, 2H); IR (neat) $\mathrm{cm}^{-1} 3064 \mathrm{w}, 2963 \mathrm{~s}, 1777 \mathrm{~s}, 1725 \mathrm{~s}, 1416 \mathrm{~s}$; mass spectrum (EI): m/e (\% relative intensity) $543(80)(\mathrm{M}+$ $\mathrm{Na})^{+} ; \mathrm{m} / \mathrm{e}$ calcd for $\mathrm{C}_{29} \mathrm{H}_{32} \mathrm{~N}_{2} \mathrm{O}_{7} \mathrm{Na} 543.2102$, found 543.2105.

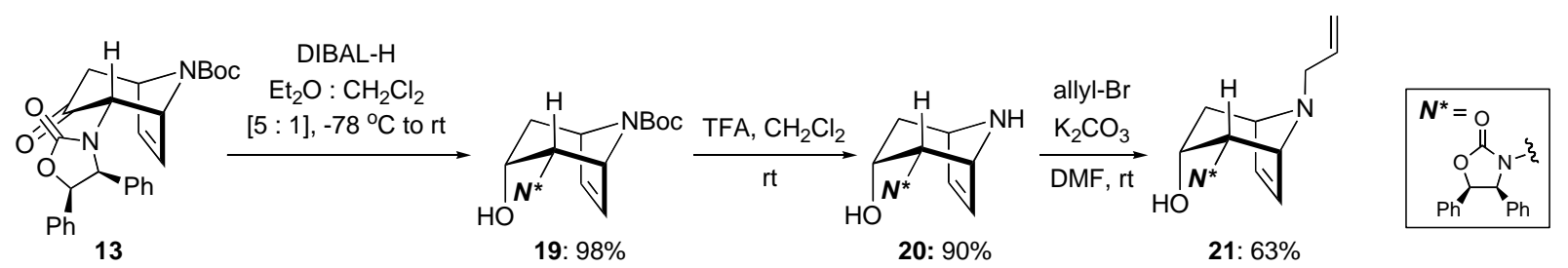

DIBAL-H Reduction. To a stirring solution of 13 [50.0 mg, $0.11 \mathrm{mmol}]$ in ether/ $\mathrm{CH}_{2} \mathrm{Cl}_{2}[0.6$ $\mathrm{mL}$, ratio $=5: 1]$ at $-78{ }^{\circ} \mathrm{C}$ was added DIBAL-H $[1.0 \mathrm{M}$ in toluene, $0.13 \mathrm{~mL}]$. The reaction was stirred at this temperature for $1 \mathrm{~h}$ before being warmed to rt over $2 \mathrm{~h}$. The reaction mixture was quenched by sat aq $\mathrm{NaHCO}_{3}$, filtered through Celite ${ }^{\mathrm{TM}}$, extracted with EtOAc [4 x $20 \mathrm{~mL}$ ]. The organic extracts were combined, washed with sat aq $\mathrm{NaCl}$, dried over $\mathrm{Na}_{2} \mathrm{SO}_{4}$, and concentrated in vacuo. Alcohol 19 was isolated as clear oil [98\%] and used without further purification in the next step.

Alcohol 19: $\mathrm{R}_{f}=0.13\left[50 \%\right.$ EtOAc in hexanes]; $[\alpha]^{23}{ }_{\mathrm{D}}=-112.0\left(\mathrm{c}=0.50, \mathrm{CH}_{2} \mathrm{Cl}_{2}\right) ;{ }^{1} \mathrm{H} \mathrm{NMR}$ $\left(400 \mathrm{MHz}, \mathrm{CDCl}_{3}\right) \delta 1.42$ (s, 9H), 1.50-1.79 (br, 1H), 1.85 (br, 1H), 2.12 (br, 0.3H), 2.31 (br, 0.7H) 2.63 (br, 0.5H), 3.10 (br, 0.5H), 4.11-4.52 (brm, 5H), 5.85 (br, 0.2H), 5.90 (d, 0.8H, J = 8.0 Hz), 6.15 (br, $0.15 \mathrm{H}), 6.34$ (br, 0.85H) 6.95-7.15 (br, 10H); IR (neat) $\mathrm{cm}^{-1} 3447 \mathrm{w}, 3055 \mathrm{w}, 2981 \mathrm{w}, 1746 \mathrm{~s}, 1691 \mathrm{~m}$; mass spectrum (APCI): m/e (\% relative intensity) $485(100)(\mathrm{M}+\mathrm{Na})^{+}$.

Boc Deprotection. To a solution of the above alcohol 19 [77.0 $\mathrm{mg}, 0.17 \mathrm{mmol}]$ in $\mathrm{CH}_{2} \mathrm{Cl}_{2}[0.7$ $\mathrm{mL}]$ at $\mathrm{rt}$ was added trifluoroacetic acid $[0.13 \mathrm{~mL}, 1.7 \mathrm{mmol}]$. The reaction was allowed to stir for $12 \mathrm{~h}$ then quenched with $10 \%$ aq $\mathrm{NaOH}$. The aqueous layer was separated and extracted with $\mathrm{CH}_{2} \mathrm{Cl}_{2}[4 \times 30$ $\mathrm{mL}$ ]. The organic extracts were combined, dried over $\mathrm{Na}_{2} \mathrm{SO}_{4}$, and concentrated in vacuo to give amine 20 as red foam that was used without further purification in the next step.

Amine 20: $\mathrm{R}_{f}=0.22\left[20 \% \mathrm{MeOH}\right.$ in $\left.\mathrm{CHCl}_{3}\right] ;[\alpha]^{23}{ }_{\mathrm{D}}=-20.6\left(\mathrm{c}=0.10, \mathrm{CHCl}_{3}\right) ;{ }^{1} \mathrm{H}$ NMR $(500$ 
$\left.\mathrm{MHz}, \mathrm{CDCl}_{3}\right) \delta 1.77(\mathrm{~d}, 1 \mathrm{H}, J=15.6 \mathrm{~Hz}), 1.89(\mathrm{ddd}, 1 \mathrm{H}, J=4.4,6.8,15.6 \mathrm{~Hz}), 3.35(\mathrm{~m}, 1 \mathrm{H}), 3.41(\mathrm{dd}$, $1 \mathrm{H}, J=4.5,7.5) 3.74(\mathrm{br}, 1 \mathrm{H}), 4.10(\mathrm{~m}, 1 \mathrm{H}), 4.38(\mathrm{~d}, 1 \mathrm{H}, J=5.2 \mathrm{~Hz}), 5.30(\mathrm{dd}, 1 \mathrm{H}, J=1.6,6.0 \mathrm{~Hz})$, $5.63(\mathrm{~d}, 1 \mathrm{H}, J=8.8 \mathrm{~Hz}), 6.16(\mathrm{dd}, 1 \mathrm{H}, J=3.6,7.6 \mathrm{~Hz}), 7.28-7.42(\mathrm{~m}, 6 \mathrm{H}), 7.47(\mathrm{~m}, 2 \mathrm{H}), 7.71(\mathrm{~m}, 2 \mathrm{H})$, (missing $\mathrm{NH}$ and $\mathrm{OH}) ;{ }^{13} \mathrm{C} \mathrm{NMR}\left(100 \mathrm{MHz}, \mathrm{CDCl}_{3}\right) \delta 35.9,59.1,60.3,65.0,66.0,68.8,81.1,82.7$, $127.4,128.9,129.1,129.5,129.6,134.7,135.7,138.5,139.3,160.8$; IR (neat) $\mathrm{cm}^{-1} 3417 \mathrm{w}, 2979 \mathrm{w}$, $1694 \mathrm{~s}, 1703 \mathrm{~m}, 1480 \mathrm{~s}$; mass spectrum (APCI): m/e (\% relative intensity) $385(100)(\mathrm{M}+\mathrm{Na})^{+}$.

$N$-Allylation. To a solution of the above amine 20 [0.15 g, $0.41 \mathrm{mmol}]$ in DMF [0.9 $\mathrm{mL}]$ at $\mathrm{rt}$ was added $\mathrm{K}_{2} \mathrm{CO}_{3}[115 \mathrm{mg}, 0.83 \mathrm{mmol}]$ and allyl bromide [0.04 $\mathrm{mL}, 0.83 \mathrm{mmol}$. The reaction mixture was allowed to stir for $12 \mathrm{~h}$ before being quenched with $\mathrm{H}_{2} \mathrm{O}[10 \mathrm{~mL}]$ and partitioned with EtOAc. The aqueous layer was extracted with EtOAc [4 x $25 \mathrm{~mL}$ ]. The combined organic layers were washed with water [6 x $25 \mathrm{~mL}$ ], then dried with $\mathrm{Na}_{2} \mathrm{SO}_{4}$, and concentrated in vacuo to give an orange oil. The crude oil was purified by silica gel flash column chromatography [gradient eluent: $0 \%$ to $50 \%$ EtOAc in hexanes] to give allylic amine 21 as white foam [63\% overall].

Allyl Amine 21: $\mathrm{R}_{f}=0.25\left[50 \%\right.$ EtOAc in hexanes]; $[\alpha]^{23}{ }_{\mathrm{D}}=-118.7\left(\mathrm{c}=0.60, \mathrm{CH}_{2} \mathrm{Cl}_{2}\right) ;{ }^{1} \mathrm{H}$ NMR $\left(500 \mathrm{MHz}, \mathrm{CDCl}_{3}\right) \delta 1.82(\mathrm{~d}, 1 \mathrm{H}, J=14.5 \mathrm{~Hz}), 2.21(\mathrm{ddd}, 1 \mathrm{H}, J=4.0,6.0,14.5 \mathrm{~Hz}), 2.62$ (br, 1H), 2.83 (br, 1H), 3.30 (br, 1H) 3.47 (br, 1H), 4.22 (br, 1H), 4.40 (dd, 1H, J = 2.5, $4.0 \mathrm{~Hz}), 5.01$ (d, $1 \mathrm{H}$, $J=10.5 \mathrm{~Hz}), 4.95(\mathrm{~d}, 1 \mathrm{H}, J=17.5 \mathrm{~Hz}), 5.32(\mathrm{~d}, 1 \mathrm{H}, J=8.0 \mathrm{~Hz}), 5.72(\mathrm{~m}, 2 \mathrm{H}), 5.88(\mathrm{~d}, 1 \mathrm{H}, J=8.0 \mathrm{~Hz})$, $6.21(\mathrm{dd}, 1 \mathrm{H}, J=2.0,4.5 \mathrm{~Hz}), 6.97(\mathrm{~m}, 4 \mathrm{H}), 7.06(\mathrm{~m}, 3 \mathrm{H}), 7.10(\mathrm{br}, 3 \mathrm{H})$, missing $-\mathrm{OH} ;{ }^{13} \mathrm{C}$ NMR $(125$ $\left.\mathrm{MHz}_{2} \mathrm{CDCl}_{3}\right) \delta 14.4,29.1,29.9,56.7,60.5,62.9,66.3,68.3,73.1,74.0,117.9,126.9,128.5,128.7$, 128.8, 128.9, 129.7, 129.9, 136.0, 138.0, 158.1; IR (neat) $\mathrm{cm}^{-1} 3110 \mathrm{w}, 3250 \mathrm{w}, 2978 \mathrm{w}, 1755 \mathrm{~s}, 1697 \mathrm{~s}$; mass spectrum (APCI): $\mathrm{m} / \mathrm{e}\left(\%\right.$ relative intensity) $425(100)(\mathrm{M}+\mathrm{Na})^{+}$.

\section{RCM APproach to PARVineostemonine.}

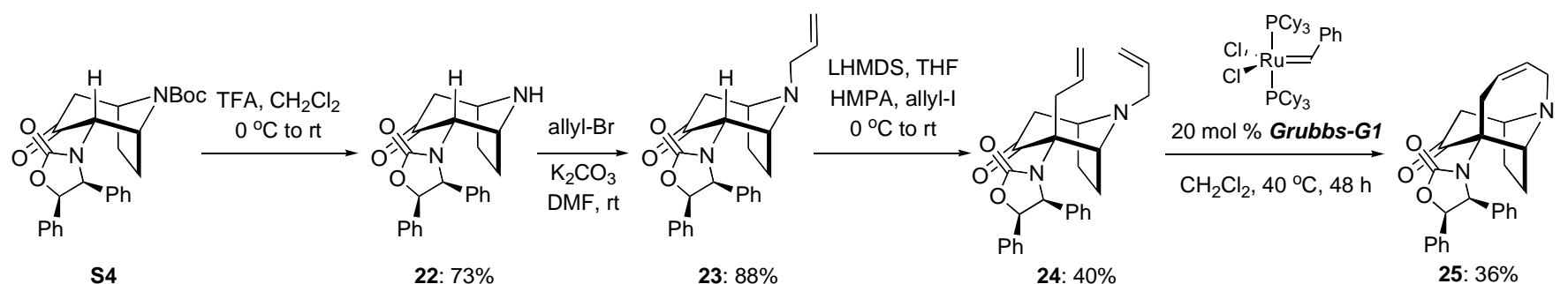

Boc Deprotection. To a solution of S4 $[250.0 \mathrm{mg}, 0.56 \mathrm{mmol}]$ in $\mathrm{CH}_{2} \mathrm{Cl}_{2}$ [1.9 $\mathrm{mL}$ ] at $\mathrm{rt}$ was added trifluoroacetic acid [0.43 $\mathrm{mL}, 5.6 \mathrm{mmol}]$. The reaction mixture was allowed to stir for $12 \mathrm{~h}$ before being quenched with $10 \%$ aq $\mathrm{NaOH}$. The aqueous layer was separated and extracted with $\mathrm{CH}_{2} \mathrm{Cl}_{2}[4 \mathrm{x}$ $30 \mathrm{~mL}$. The organic extracts were combined, dried over $\mathrm{Na}_{2} \mathrm{SO}_{4}$, and concentrated in vacuo to give amine 22 as yellow foam [ $73 \%$ ] that was used without further purification in the next step.

Amine 22: $\mathrm{R}_{f}=0.55\left[5 \% \mathrm{MeOH}\right.$ in $\left.\left.\mathrm{CHCl}_{3}\right] ; \alpha\right]^{23}{ }_{\mathrm{D}}=11.3\left(\mathrm{c}=1.90, \mathrm{CHCl}_{3}\right) ;{ }^{1} \mathrm{H} \mathrm{NMR}(400$ $\left.\mathrm{MHz}, \mathrm{CDCl}_{3}\right) \delta 1.08(\mathrm{~m}, 1 \mathrm{H}), 1.30(\mathrm{~m}, 1 \mathrm{H}), 1.56(\mathrm{~m}, 1 \mathrm{H}), 1.69(\mathrm{~m}, 1 \mathrm{H}), 2.46(\mathrm{dd}, 1 \mathrm{H}, J=2.0,15.2 \mathrm{~Hz})$, $2.63(\mathrm{dd}, 1 \mathrm{H}, J=5.6,16 \mathrm{~Hz}), 3.63(\mathrm{dd}, 1 \mathrm{H}, J=3.4,6.8 \mathrm{~Hz}), 3.82(\mathrm{t}, 1 \mathrm{H}, J=5.6 \mathrm{~Hz}), 4.87(\mathrm{~d}, 1 \mathrm{H}, J=$ 
$4.0 \mathrm{~Hz}), 5.17(\mathrm{~d}, 1 \mathrm{H}, J=7.6 \mathrm{~Hz}), 6.04(\mathrm{~d}, 1 \mathrm{H}, J=8.0 \mathrm{~Hz}), 6.95-7.13(\mathrm{~m}, 10 \mathrm{H}),(\operatorname{missing~NH}) ;{ }^{13} \mathrm{C} \mathrm{NMR}$ $\left(100 \mathrm{MHz}, \mathrm{CDCl}_{3}\right) \delta 26.7,29.9,30.6,50.6,56.3,58.9,63.2,68.2,81.7,118.4,126.5,128.0,128.2$, 128.4, 134.2, 136.5, 159.1, 203.7; IR (neat) $\mathrm{cm}^{-1} 3050 \mathrm{w}, 2963 \mathrm{w}, 2953 \mathrm{~s}, 1746 \mathrm{~s}, 1725 \mathrm{~s}$; mass spectrum (APCI): $\mathrm{m} / \mathrm{e}\left(\%\right.$ relative intensity) $385(90)(\mathrm{M}+\mathrm{Na})^{+} ; \mathrm{m} / \mathrm{e}$ calcd for $\mathrm{C}_{22} \mathrm{H}_{22} \mathrm{~N}_{2} \mathrm{O}_{3} \mathrm{Na} 385.1523$, found 385.1493.

$N$-Allylation. To a solution of the above amine $22[0.103 \mathrm{~g}, 0.28 \mathrm{mmol}]$ in DMF $[0.9 \mathrm{~mL}]$ at $\mathrm{rt}$ was added $\mathrm{K}_{2} \mathrm{CO}_{3}[79.0 \mathrm{mg}, 0.57 \mathrm{mmol}]$ then allyl bromide $[0.027 \mathrm{~mL}, 0.31 \mathrm{mmol}]$. The reaction was allowed to stir for $12 \mathrm{~h}$ then quenched with water $[10 \mathrm{~mL}]$ and partitioned with EtOAc. The aqueous layer was extracted with EtOAc [4 x $25 \mathrm{~mL}$ ]. The combined organic layers were washed with water [6 x $25 \mathrm{~mL}$ ], then dried with $\mathrm{Na}_{2} \mathrm{SO}_{4}$, and concentrated in vacuo to give an orange oil. The crude oil was purified by silica gel column chromatography [gradient eluent: $0 \%$ to $50 \%$ EtOAc in hexanes] to give allyl amine 23 [88\%] as white foam.

Allyl Amine 23: $\mathrm{R}_{f}=0.20\left[50 \%\right.$ EtOAc in hexanes]; $[\alpha]^{23}{ }_{\mathrm{D}}=-25.0\left(\mathrm{c}=0.20, \mathrm{CH}_{2} \mathrm{Cl}_{2}\right) ;{ }^{1} \mathrm{H}$ NMR (400 MHz, $\left.\mathrm{CDCl}_{3}\right) \delta 1.11-1.38(\mathrm{~m}, 2 \mathrm{H}), 1.50(\mathrm{~m}, 1 \mathrm{H}), 1.84(\mathrm{~m}, 1 \mathrm{H}), 2.32(\mathrm{dd}, 1 \mathrm{H}, J=1.8,15.2$ $\mathrm{Hz}), 2.73(\mathrm{dd}, 1 \mathrm{H}, J=4.0,15.2 \mathrm{~Hz}), 3.19$ (dd, 1H, $J=4.8,6.4 \mathrm{~Hz}), 3.33$ (brm, 1H), 3.49 (brm, 1H), 5.02 $(\mathrm{d}, 1 \mathrm{H}, J=2.9 \mathrm{~Hz}), 5.10-5.23(\mathrm{~m}, 3 \mathrm{H}), 5.79-5.91(\mathrm{~m}, 2 \mathrm{H}), 6.03(\mathrm{~d}, 1 \mathrm{H}, J=7.6 \mathrm{~Hz}), 6.92-7.14(\mathrm{~m}, 10 \mathrm{H})$; ${ }^{13} \mathrm{C}$ NMR $\left(125 \mathrm{MHz}, \mathrm{CDCl}_{3}\right) \delta 25.4,28.3,53.8,59.5,62.8,63.4,64.7,81.7,117.8,126.5,127.2,128.1$, $128.2,128.3,128.4,129.3,134.4,135.7,136.6,159.2,205.0$; IR (neat) $\mathrm{cm}^{-1} 2989 \mathrm{w}, 2926 \mathrm{w}, 1746 \mathrm{~s}$, $1691 \mathrm{~s}, 1477 \mathrm{~s}$; mass spectrum (APCI): m/e (\% relative intensity) $403(60)(\mathrm{M}+\mathrm{H})^{+} ; \mathrm{m} / \mathrm{e}$ calcd for $\mathrm{C}_{25} \mathrm{H}_{27} \mathrm{~N}_{2} \mathrm{O}_{3}$ 403.2016, found 403.2003.

Allylation. To a solution of the above allyl amine 23 [103 $\mathrm{mg}, 0.26 \mathrm{mmol}]$ in THF [1 $\mathrm{mL}]$ was added HMPA [0.05 mL, $0.31 \mathrm{mmol}$. The solution was cooled to $0{ }^{\circ} \mathrm{C}$ and LHMDS [0.61 $\mathrm{mL}, 0.5 \mathrm{M}$, $0.31 \mathrm{mmol}]$ was added, and after stirring for $30 \mathrm{~min}$, allyl iodide [0.04 mL, $0.39 \mathrm{mmol}]$ was added and the resulting mixture allowed to warm to $\mathrm{rt}$ and was stirred for $12 \mathrm{~h}$. The reaction was quenched with sat aq $\mathrm{NaHCO}_{3}$, extracted with EtOAc [4 x $25 \mathrm{~mL}$ ], and the combined organic fractions were dried over $\mathrm{Na}_{2} \mathrm{SO}_{4}$, filtered, and concentrated in vacuo. The crude oil was purified by silica gel flash column chromatography [6\% EtOAc in hexanes] to give diene 24 [40\%] yield as yellow oil.

Diene 24: $\mathrm{R}_{f}=0.73\left[50 \%\right.$ EtOAc in hexanes]; $[\alpha]^{23}{ }_{\mathrm{D}}=-13.0\left(\mathrm{c}=0.20, \mathrm{CH}_{2} \mathrm{Cl}_{2}\right) ;{ }^{1} \mathrm{H}$ NMR $(500$ $\left.\mathrm{MHz}, \mathrm{CDCl}_{3}\right) \delta 1.07(\mathrm{~m}, 1 \mathrm{H}), 1.51(\mathrm{~m}, 1 \mathrm{H}), 1.83(\mathrm{~m}, 3 \mathrm{H}), 2.69(\mathrm{dd}, 1 \mathrm{H}, J=6.0,17.5 \mathrm{~Hz}), 3.00(\mathrm{~m}, 2 \mathrm{H})$, $3.06(\mathrm{dd}, 1 \mathrm{H}, J=6.5,13.5 \mathrm{~Hz}), 3.14(\mathrm{dd}, 1 \mathrm{H}, J=8.5,14.5 \mathrm{~Hz}), 3.28(\mathrm{t}, 1 \mathrm{H}, J=5.5 \mathrm{~Hz}), 4.34(\mathrm{~d}, 1 \mathrm{H}, J=$ $6.0 \mathrm{~Hz}), 5.11-5.23(\mathrm{~m}, 4 \mathrm{H}), 5.70(\mathrm{~d}, 1 \mathrm{H}, J=8.0 \mathrm{~Hz}), 5.87(\mathrm{~d}, 1 \mathrm{H}, J=8.0 \mathrm{~Hz}), 5.82-5.92(\mathrm{~m}, 2 \mathrm{H}), 6.97-$ $7.14(\mathrm{~m}, 10 \mathrm{H}) ;{ }^{13} \mathrm{C} \mathrm{NMR}\left(75 \mathrm{MHz}, \mathrm{CDCl}_{3}\right) \delta 23.9,24.0,27.5,27.8,29.9,40.8,57.0,58.4,58.7,66.2$, 72.4, 80.4, 117.4, 119.4, 126.0, 127.9, 129.3, 133.3, 135.0, 136.4, 136.9, 157.6, 206.0 (missing 1 carbon due to overlap); IR (neat) $\mathrm{cm}^{-1} 2990 \mathrm{w}, 2963 \mathrm{w}, 1777 \mathrm{~s}, 1725 \mathrm{~s}, 1416 \mathrm{~s}$; mass spectrum (APCI): m/e (\% relative intensity) $443(90)(\mathrm{M}+\mathrm{H})^{+} ; \mathrm{m} / \mathrm{e}$ calcd for $\mathrm{C}_{28} \mathrm{H}_{31} \mathrm{~N}_{2} \mathrm{O}_{3} 443.2329$, found 443.2332 .

RCM. To a solution of the above diene 24 [ $7 \mathrm{mg}, 0.016 \mathrm{mmol}$ ] in $0.5 \mathrm{~mL}$ of $\mathrm{CH}_{2} \mathrm{Cl}_{2}$ was added 
Grubbs'-G1 (1.3 mg, $0.002 \mathrm{mmol})$. The reaction was heated to $40{ }^{\circ} \mathrm{C}$ for $1 \mathrm{~d} .{ }^{1} \mathrm{H}$ NMR showed some starting material and $10 \mathrm{~mol} \%$ more catalyst was added to the reaction and heating continued for $1 \mathrm{~d}$ until no starting material was seen. The reaction was concentrated in vacuo and purified by silica gel column chromatography [gradient eluent $0 \%$ to $30 \% \mathrm{MeOH}$ in $\mathrm{CHCl}_{3}$ ] to give aza-tricycle 25 [36\%] as yellow oil.

Aza-Tricycle 25: $\mathrm{R}_{f}=0.40\left[20 \% \mathrm{MeOH}\right.$ in $\left.\mathrm{CHCl}_{3}\right] ;[\alpha]^{23}{ }_{\mathrm{D}}=-16.0\left(\mathrm{c}=0.20, \mathrm{CH}_{2} \mathrm{Cl}_{2}\right) ;{ }^{1} \mathrm{H} \mathrm{NMR}$ $\left(400 \mathrm{MHz}, \mathrm{CDCl}_{3}\right) \delta 1.43(\mathrm{~m}, 2 \mathrm{H}), 1.75(\mathrm{~m}, 2 \mathrm{H}), 2.63(\mathrm{dd}, 1 \mathrm{H}, J=8.8,13.2 \mathrm{~Hz}), 2.75(\mathrm{dd}, 1 \mathrm{H}, J=5.2$, $18.0 \mathrm{~Hz}$ ), 3.49 (brs, 1H), 3.59 (dd, 1H, $J=6.8,13.0 \mathrm{~Hz}), 3.64$ (d, 1H, J=6.4 Hz), 3.72-3.77 (brm, 1H), $3.79(\mathrm{~m}, 1 \mathrm{H}), 3.94(\mathrm{~d}, 1 \mathrm{H}, J=4.4 \mathrm{~Hz}), 5.11(\mathrm{~d}, 1 \mathrm{H}, J=8.4 \mathrm{~Hz}), 5.29(\mathrm{~m}, 1 \mathrm{H}), 5.83(\mathrm{~m}, 1 \mathrm{H}), 5.90(\mathrm{~d}, 1 \mathrm{H}$, $J=8.0 \mathrm{~Hz}), 6.93-7.14(\mathrm{~m}, 10 \mathrm{H}) ;{ }^{13} \mathrm{C} \mathrm{NMR}\left(125 \mathrm{MHz}, \mathrm{CDCl}_{3}\right) \delta 26.6,27.2,28.0,29.9,30.2,35.4,35.9$, 47.6, 58.9, 63.8, 81.6, 126.1, 126.3, 128.1, 132.2, 132.3, 132.4, 133.7, 134.3, 136.6, 157.6, 205.7; IR (neat) $\mathrm{cm}^{-1} 2989 \mathrm{~s}, 2926 \mathrm{~m}, 1746 \mathrm{~s}, 1477 \mathrm{w}$; mass spectrum (APCI): m/e (\% relative intensity) 415 (60) (M $+\mathrm{H})^{+} ; \mathrm{m} / \mathrm{e}$ calcd for $\mathrm{C}_{26} \mathrm{H}_{27} \mathrm{~N}_{2} \mathrm{O}_{3} 415.2016$, found 415.2013.

\section{RETRO-MANNICH From the FoOTNOTE.}

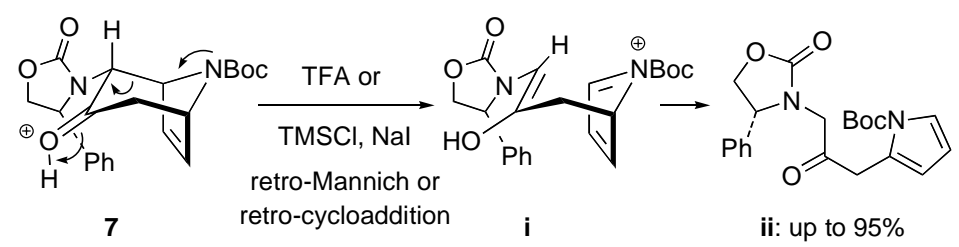

To a solution of cycloadduct $7[28.0 \mathrm{mg}, 0.075 \mathrm{mmol}]$ in $\mathrm{CH}_{2} \mathrm{Cl}_{2}$ [0.5 mL] was added trifluoroacetic acid $\left[0.060 \mathrm{~mL}, 0.75 \mathrm{mmol}\right.$ ) at $0{ }^{\circ} \mathrm{C}$. After $12 \mathrm{~h}$, TLC showed no starting material was left. The reaction was quenched with $10 \%$ aq $\mathrm{NaOH}$. The aqueous layer was then separated and extracted with $\mathrm{CH}_{2} \mathrm{Cl}_{2}[3 \times 20 \mathrm{~mL}]$. The organic extracts were combined, dried over $\mathrm{NaSO}_{4}$, and concentrated in vacuo to give pyrrole ii [95\%] as red foam.

Pyrrole ii: $\mathrm{R}_{f}=0.52\left[50 \%\right.$ EtOAc in Hexanes]; $[\alpha]^{23} \mathrm{D}=-135.0\left(\mathrm{c}=0.40, \mathrm{CHCl}_{3}\right) ;{ }^{1} \mathrm{H}$ NMR $\left(400 \mathrm{MHz}, \mathrm{CDCl}_{3}\right) \delta 1.50(\mathrm{~s}, 9 \mathrm{H}), 3.55(\mathrm{~d}, 1 \mathrm{H}, J=18.4 \mathrm{~Hz}), 3.76(\mathrm{~d}, 1 \mathrm{H}, J=17.6 \mathrm{~Hz}), 3.85(\mathrm{~d}, 1 \mathrm{H}, J=$ 17.6), $4.14(\mathrm{dd}, 1 \mathrm{H}, J=7.2,8.8 \mathrm{~Hz}) 4.42(\mathrm{~d}, 1 \mathrm{H}, J=18.4 \mathrm{~Hz}), 4.70(\mathrm{t}, 1 \mathrm{H}, J=8.8 \mathrm{~Hz}), 5.04(\mathrm{dd}, 1 \mathrm{H}, J=$ 7.2, $8.8 \mathrm{~Hz}), 6.06$ (brm, 1H), $6.10(\mathrm{t}, 1 \mathrm{H}, J=3.2 \mathrm{~Hz}), 7.15(\mathrm{dd}, 1 \mathrm{H}, J=2.0,3.6 \mathrm{~Hz}), 7.28(\mathrm{~m}, 2 \mathrm{H}) 7.35-$ $7.42(\mathrm{~m}, 3 \mathrm{H}) ;{ }^{13} \mathrm{C} \mathrm{NMR}\left(100 \mathrm{MHz}, \mathrm{CDCl}_{3}\right) \delta 28.1,40.3,49.0,50.3,60.1,70.4,84.2,110.6,115.2$, 121.8, 126.7, 127.4, 129.3, 129.5, 149.6, 137.7, 201.6; IR (neat) $\mathrm{cm}^{-1} 3000 \mathrm{w}, 2977 \mathrm{w}, 1697 \mathrm{~s}, 1703 \mathrm{~s}$; mass spectrum (APCI): m/e (\% relative intensity) 407 (100) $(\mathrm{M}+\mathrm{Na})^{+} ; \mathrm{m} / \mathrm{e}$ calcd for $\mathrm{C}_{16} \mathrm{H}_{16} \mathrm{~N}_{2} \mathrm{O}_{3}(\mathrm{M}-$ Boc + H) 284.1155, found 284.1139. 